Aequationes Mathematicae, to appear.

\title{
SOLVING FUNCTIONAL EQUATIONS VIA FINITE SUBSTITUTIONS
}

\author{
MIHÁLY BESSENYEI AND CSABA G. KÉZI \\ Dedicated to the 60th birthday of Professor László Székelyhidi
}

\begin{abstract}
In this paper, we study single variable functional equations that involve one unknown function and a finite set of known functions that form a group under the operation of composition. The main theorems give sufficient conditions for the existence and uniqueness of a (local) solution and also stability-type result for the solution. In the proofs, beside the standard methods of classical analysis, some group theoretical tools play a key role.
\end{abstract}

\section{INTRODUCTION}

Assume that $F, h$ further $g_{1}, \ldots, g_{r}$ are given functions (with proper ranges and domains) such that the letter ones form a group under the operation of composition. The investigations of this note are focused on the single variable functional equation (with unknown function $f$ ) given by

$$
F\left(f \circ g_{1}(t), \ldots, f \circ g_{r}(t)\right)=h(t) .
$$

We make no attempt here to give an exhaustive account of the background of this equation, just mention that it is motivated by some results of Babbage [2], [3] and was studied by several authors. Presić investigated the case when $F$ is supposed to be linear [15] (for its English version, see [12] or [14]). A nonlinear version is also due to him [16]. The key idea of these papers is to create a system of equations from (1) via substitutions. This method works in other settings and can be combined by Cramer's Rule and the Inverse Function Theorem [5], by the Implicit Function Theorem [6], and by some group theoretical results [7]. It also worth mentioning that a special branch of (1) has found its place in competition problems for secondary school students: the books of Brodskii-Slipenko [8] and of Small [18] contain a rich material to demonstrate this fact.

The aim of the present paper is to give a local existence and uniqueness theorem for the solution of (1). Applying substitutions, a system of nonlinear equations can be derived. Under suitable regularities, the Inverse Function Theorem guarantees representation for the only possible (local) solution $f$. However, the so-called compatibility equations should also be checked (for precise details, consult [5]). This process can be done using the Orbit Stabilizer Lemma and the central property of a suitable stabilizer. The Orbit Stabilizer Lemma is also crucial in determining the number of initial values for (1) such as in omitting the fixed point condition used in the second theorem of [5].

Date: Submitted: may 23, 2012; accepted: october 23, 2012.

2010 Mathematics Subject Classification. Primary 39B12, Secondary 20F38, 26B10, 26 B12.

Key words and phrases. Functional equations of a single variable; implicit functions; finite groups; cosets of finite function groups; permutations of matricies.

This research has been supported by the Hungarian Scientific Research Fund (OTKA) Grants NK-81402. 


\section{THE MAIN RESULT}

In the sequel, $G(H)$ stands for a finite set of functions with domain and range $H$ (where $H$ is a nonempty subset of the reals), such that the functions form a group under the operation of composition. Assume $G(H)=\left\{g_{i} \mid i \in \Gamma\right\}$ with some finite set $\Gamma$. For our convenience, define the operation $*$ on $\Gamma$ via the conventions $i * j=k$ if and only if $g_{i} g_{j}=g_{k}$ holds. Then $(\Gamma, *)$ is a finite group isomorphic to $(G(H), \circ)$; the inverse of $i \in \Gamma$ is denoted by $i^{-1}$.

As usual, if a matrix $A$ is given, then $a_{i j}$ denotes the common element of the $i$ th row and $j$ th column. We shall use the notation $\mathcal{M}_{r}$ for the set of quadratic matrices of type $r \times r$. If $A$ is a quadratic matrix and $\pi$ is a permutation on its type set, then $A_{\pi}$ stands for the matrix which is obtained by permuting the rows and then the columns (or equivalently: the columns and then the rows) of $A$ by $\pi$. Similarly, if a vector $B$ is given, then $B_{\pi}$ denotes the vector obtained by arranging the components of $B$ by $\pi$. In our investigations, $\pi$ shall be considered as $* i$ with suitable $(\Gamma, *)$.

The next lemma plays a crucial role in the proof of the Main Theorem. It is known as the Orbit Stabilizer Lemma, and can be found in any textbook on permutation groups (consult, for example, [9] or [11]). For the Reader's convenience, we present here its proof.

Lemma. If $H \subset \mathbb{R}$ is a nonempty set, $G(H)$ is a group of functions and $\xi \in H$ is arbitrary, then $G_{\xi}(H)=\{g \in G(H) \mid g(\xi)=\xi\}$ is a subgroup of $G(H)$. Consider the left cosets of $G_{\xi}(H)$ in $G(H)$. Then, the elements $g$ and $h$ of $G(H)$ belong to the same left coset if and only if $g(\xi)=h(\xi)$.

Proof. If $g, h \in G_{\xi}(H)$ are arbitrary, then $g(\xi)=h(\xi)=\xi$, thus both $g \circ h(\xi)=\xi$ and $g^{-1}(\xi)=\xi$ hold. That is, $G_{\xi}(H)$ is closed under the group operations, and hence is indeed a subgroup. For proving the second statement, first take elements $h_{1}$ and $h_{2}$ belonging to the coset $h G_{\xi}$. Then we have the representations $h_{1}=h \circ g_{1}$ and $h_{2}=h \circ g_{2}$ with suitable $g_{1}, g_{2} \in G_{\xi}$; hence

$$
h_{1}(\xi)=h \circ g_{1}(\xi)=h(\xi)=h \circ g_{2}(\xi)=h_{2}(\xi) .
$$

Assume conversely, that $h_{1}(\xi)=h_{2}(\xi)$ and set $g:=h_{1}^{-1} \circ h_{2}$. Then, $g \in G_{\xi}$ which implies that $h_{2} \in h_{1} G_{\xi}$, and the proof is completed.

For a fixed $\xi \in H$, the set $G_{\xi}(H)$ is called the stabilizer of $\xi$ in $G(H)$. In view of the previous lemma, the stabilizer of an arbitrary element of $H$ is a subgroup of $G(H)$. Assume that the stabilizer $G_{\xi}(H)$ of $\xi$ in $G(H)$ has $m$ elements and is given by

$$
G_{\xi}(H)=\left\{g_{k n+1} \mid k=0, \ldots, m-1\right\} .
$$

As the previous lemma shows, the stabilizer is a subgroup; let $\left\{g_{1}, \ldots, g_{n}\right\}$ be a representation set of $G(H)$ with respect to $G_{\xi}(H)$. By Lagrange's theorem, $|G(H)|=m n$. Moreover, without loss of generality, we may assume that the elements of $G(H)$ are indexed according to the following table.

\begin{tabular}{|c||cccccc|}
\hline$\circ$ & $g_{1}$ & $g_{n+1}$ & $\ldots$ & $g_{k n+1}$ & $\ldots$ & $g_{(m-1) n+1}$ \\
\hline \hline$g_{1}$ & $g_{1}$ & $g_{n+1}$ & $\ldots$ & $g_{k n+1}$ & $\ldots$ & $g_{(m-1) n+1}$ \\
$g_{2}$ & $g_{2}$ & $g_{n+2}$ & $\ldots$ & $g_{k n+2}$ & $\ldots$ & $g_{(m-1) n+2}$ \\
$\vdots$ & $\vdots$ & $\vdots$ & $\ddots$ & $\vdots$ & $\ddots$ & $\vdots$ \\
$g_{l}$ & $g_{l}$ & $g_{n+l}$ & $\ldots$ & $g_{k n+l}$ & $\ldots$ & $g_{(m-1) n+l}$ \\
$\vdots$ & $\vdots$ & $\vdots$ & $\ddots$ & $\vdots$ & $\ddots$ & $\vdots$ \\
$g_{n}$ & $g_{n}$ & $g_{2 n}$ & $\ldots$ & $g_{(k+1) n}$ & $\ldots$ & $g_{m n}$ \\
\hline
\end{tabular}


Evaluating all elements at $\xi$, the values obtained in a particular row coincide (that is, they form a left coset of $G_{\xi}(H)$ ) while the values in a particular column are pairwise distinct. Moreover, for all $l=1, \ldots, n$ and $k=0, \ldots, m-1$ we have

$$
l *(k n+1)=k n+l .
$$

In these settings, due to the Orbit Stabilizer Lemma again, $g_{i}(\xi)=g_{j}(\xi)$ if and only if $i \equiv j$ $(\bmod n)$. Hence if we require initial value conditions for the unknown $f$, then we have to take into consideration the number of left cosets induced by the stabilizer: The number of conditions must divide the order of the group $G(H)$. Therefore, in the rest of the paper, (1) shall be studied in the form

$$
\begin{aligned}
F\left(f \circ g_{1}(t), \ldots, f \circ g_{m n}(t)\right) & =h(t), \\
f \circ g_{k n+l}(\xi) & =\eta_{l} .
\end{aligned}
$$

The Main Result of the paper is presented in the following theorem. In the proof, beside the Orbit Stabilizer Lemma, the Inverse Function Theorem is applied (see [17]). In fact, this theorem provides the only possible (local) solution $f$. The assumption that the stabilizer is a subset of the central $Z(G(H))$ of the group guarantees compatibility, in other words, that $f$ is indeed a solution.

Theorem 1. Let $H \subset \mathbb{R}$ be a nonempty open set, $\xi \in H$, and $G(H)=\left\{g_{1}, \ldots, g_{m n}\right\}$ be a group of continuous functions such that $g_{i}(\xi)=g_{j}(\xi)$ if and only if $i \equiv j(\bmod n)$. Let $\eta \in \mathbb{R}^{n}, p=$ $(\eta, \ldots, \eta) \in \mathbb{R}^{m n}$ and let $h: H \rightarrow \mathbb{R}$ be a continuous function, $F: \mathbb{R}^{m n} \rightarrow \mathbb{R}$ be a continuously differentiable function such that $F\left(p_{* i}\right)=h \circ g_{i}(\xi)$ for all $i=1, \ldots, m n$. Define the mapping $A: \mathbb{R}^{m n} \rightarrow \mathcal{M}_{m n}$ by

$$
A(x):=\left[\partial_{j * i-1} F\left(x_{* i}\right)\right] .
$$

If $A$ is regular at $p$ and $G_{\xi}(H) \subset Z(G(H))$, then there exist a $G(H)$-invariant open set $H_{0} \subset H$ containing $\xi$ and a unique differentiable function $f: H_{0} \rightarrow \mathbb{R}$ satisfying (4).

Proof. By the Orbit Stabilizer Lemma, the assumptions $g_{i}(\xi)=g_{j}(\xi)$ if and only if $i \equiv j(\bmod n)$ imply that the stabilizer $G_{\xi}(H)$ is of order $m$. Therefore, without loss of generality we may assume that the elements of $G(H)$ are listed as in (3), where $G_{\xi}(H)$ is given by (2) and the representation set of the left cosets of $G_{\xi}(H)$ in $G(H)$ is $\left\{g_{1}, \ldots, g_{n}\right\}$. Denote the function whose components in order are $h \circ g_{1}, \ldots, h \circ g_{m n}$ by $h_{g}$, and define the mapping $\Psi: \mathbb{R}^{m n} \rightarrow \mathbb{R}^{m n}$ by the formula

$$
\Psi(x):=\left[F\left(x_{* i}\right)\right] .
$$

Then, $F\left(p_{* i}\right)=h \circ g_{i}(\xi)$ implies $\Psi(p)=h_{g}(\xi)$. Since the mapping $\Psi$ is continuously differentiable, the definition of the operation $*$ and the Chain Rule give

$$
\Psi^{\prime}(p)=\left[\partial_{j * i^{-1}} F\left(p_{* i}\right)\right]=A(p) .
$$

The last term is nonsingular by assumption, hence the Inverse Function Theorem guarantees the existence of neighborhoods $U$ of $p$ and $V$ of $h_{g}(\xi)$ such that $\Psi: U \rightarrow V$ is a local diffeomorphism. The continuity of $h$ and $g_{k}$ guarantees the existence of an open interval $I$ containing $\xi$ such that $h_{g}(t) \in V$ whenever $t \in I$. Since $g_{i}(I)=g_{j}^{-1}(I)$ with $j=i^{-1}$ and the elements of $G(H)$ are continuous, the sets $g_{i}(I)$ are open. Set

$$
H_{0}:=\bigcup_{l=1}^{n} \bigcap_{k=0}^{m-1} g_{k n+l}(I) .
$$


Then $H_{0}$ is a nonempty, open subset of $I$ containing $\xi$ and, by construction, is invariant under the action of the elements $G(H)$. For simplicity we may assume that $H_{0}$ has exactly $n$ components, that is, the set $\cap_{k=0}^{m-1} g_{k n+l}(I)$ are pairwise disjoint for all $l=1, \ldots, n$.

Define now the function $f: H_{0} \rightarrow \mathbb{R}$ given by $f(t):=f_{i} \circ g_{i}^{-1}(t)$ provided $t \in g_{i}(I)$, where

$$
f_{i}:=\operatorname{pr}_{i} \circ \Psi^{-1} \circ h_{g}
$$

and $\mathrm{pr}_{i}$ denotes the $i$ th projection of $\mathbb{R}^{m n}$ onto $\mathbb{R}$. We shall verify that the set of the functions $\left\{f_{i} \mid i=1, \ldots, m n\right\}$ are compatible. To do this, it suffice to check that $f_{k n+l}=f_{l} \circ g_{k n+1}$. Consider on $I$ the rearranged form $h_{g}=\Psi\left(f_{1}, \ldots, f_{m n}\right)$ of the definition of $f_{i}$. Since $G_{\xi}(H)$ belongs to the central of $G(H)$,

$$
\begin{aligned}
h \circ g_{l} \circ g_{k n+1} & =h \circ g_{l *(k n+1)} \\
& =\Psi_{l *(k n+1)}\left(f_{1}, \ldots, f_{m n}\right) \\
& =F\left(f_{1 * l *(k n+1)}, \ldots, f_{m n * l *(k n+1)}\right) \\
& =F\left(f_{1 *(k n+1) * l}, \ldots, f_{m n *(k n+1) * l}\right) .
\end{aligned}
$$

That is,

$$
h_{g} \circ g_{k n+1}=\Psi\left(f_{1 *(k n+1)}, \ldots, f_{m n *(k n+1)}\right)
$$

holds on $I$. Furthermore, the internal function of the right-hand side is continuous on $I$, and hence there exists a neighborhood of $\xi$ such that $\left(f_{1 * l}(t), \ldots, f_{m n * l}(t)\right)$ belongs to $U$ whenever $t$ is taken from this neighborhood. For simplicity we may assume that this neighborhood is exactly $I$. (Otherwise take the nonempty intersection of the neighborhood with $I$.) Then the identity above can be written as

$$
\Psi^{-1} \circ h_{g} \circ g_{k n+1}=\left(f_{1 *(k n+1)}, \ldots, f_{n *(k n+1)}\right) .
$$

The $l$ th projection of the left-hand side is, by definition, $f_{l} \circ g_{k n+1}$, while the $l$ th projection of the right-hand side is exactly $f_{l *(k n+1)}=f_{k n+l}$, verifying the desired compatibility. To complete the proof, observe that the first component of the equation $\Psi\left(f_{1}, \ldots, f_{m n}\right)=h_{g}$ leads to (4).

The algebraic assumptions immediately arise the natural question whether which finite groups can be represented as a group of functions over a suitable subset of the reals. It turns out (see [7]) that every finite group has a representation as continuous functions acting on an open set.

Let us note that Theorem 1 generalize considerably the second Theorem of [5]: The existence on a common fixed point of the elements of $G(H)$, similarly to the Abelian structure of $G(H)$ is dropped now. Also, the regularity assumptions of Theorem 1 are weaker than that of the second Corollary of [7]; however, in the latter result, there is no further algebraic restriction on the stabilizer.

Assume now that the left-hand side of (1) is linear, that is, beside the usual assumptions, let $\alpha_{1}, \ldots, \alpha_{n}: H \rightarrow \mathbb{R}$ be given functions and consider the functional equation

$$
\sum_{k=1}^{n} \alpha_{k}(t) f \circ g_{k}(t)=h(t) .
$$

In this setting, under some reasonable requirements, there is no further need for initial value conditions and the existence of a unique global solution $f$ can be verified. Moreover, an error estimation can also be given for functions fulfilling (5) only "approximately". 
Theorem 2. Let $H \subset \mathbb{R}$ be a nonempty set, $G(H):=\left\{g_{1}, \ldots, g_{n}\right\}$ be a group functions and let $\alpha_{1}, \ldots, \alpha_{n} ; h: H \rightarrow \mathbb{R}$ be given functions. Assume that the determinant of the matrix

$$
A:=\left[\alpha_{j * i^{-1}} \circ g_{i}\right]
$$

is nonvanishing on $H$. If $A_{h}$ stands for the matrix which is obtained from $A$ replacing the first column by, in turn, $h \circ g_{1}, \ldots, h \circ g_{n}$, then $f: H \rightarrow \mathbb{R}$ given by $f=\operatorname{det} A_{h} / \operatorname{det} A$ is the unique solution of (5) on $H$. Moreover, if $\varphi: H \rightarrow \mathbb{R}$ satisfies the inequality

$$
\left|\sum_{k=1}^{n} \alpha_{k}(t) \varphi \circ g_{k}(t)-h(t)\right| \leq \varepsilon,
$$

then

$$
|f(t)-\varphi(t)| \leq \varepsilon\left\|A^{-1}(t)\right\|_{\infty}
$$

Proof. For the first statement, see the first Theorem of [5]. For the second one, consider the function $\varepsilon: H \rightarrow \mathbb{R}$ defined by

$$
\varepsilon(t):=\sum_{k=1}^{n} \alpha_{k}(t) \varphi \circ g_{k}(t)-h(t) .
$$

Then, $\varphi$ satisfies (5) with $h+\varepsilon$ on the right hand-side instead of $h$, and the stability inequality on $\varphi$ yields $|\varepsilon(t)| \leq \varepsilon$ for all $t \in H$. Moreover, due to the first part of the theorem, we have the representations

$$
f(t)=\frac{\operatorname{det} A_{h}(t)}{\operatorname{det} A(t)}, \quad \varphi(t)=\frac{\operatorname{det} A_{h+\varepsilon}(t)}{\operatorname{det} A(t)},
$$

where, for a given function $g: H \rightarrow \mathbb{R}$, the symbol $A_{g}$ stands for the matrix which can be obtained from $A$ replacing the first column by, in turn, $g \circ g_{1}, \ldots, g \circ g_{n}$. Applying the linearity of the mapping $g \rightarrow \operatorname{det} A_{g}$, expanding the expression obtained, using the representation of the inverse of a square matrix, and, at last, considering the definition of $\|\cdot\|_{\infty}$, we get

$$
\begin{aligned}
|f(t)-\varphi(t)| & =\frac{\left|\operatorname{det} A_{h}(t)-\operatorname{det} A_{h+\varepsilon}(t)\right|}{|\operatorname{det} A(t)|}=\frac{\left|\operatorname{det} A_{\varepsilon}(t)\right|}{|\operatorname{det} A(t)|} \\
& =\frac{1}{|\operatorname{det} A(t)|}\left|\sum_{k=1}^{n}(-1)^{k+1} \operatorname{det} A_{1, k}(t) \cdot \varepsilon \circ g_{k}(t)\right| \\
& \leq \frac{1}{|\operatorname{det} A(t)|} \sum_{k=1}^{n}\left|\varepsilon \circ g_{k}(t)\right| \cdot\left|(-1)^{k+1} \operatorname{det} A_{1, k}(t)\right| \\
& \leq \varepsilon \sum_{k=1}^{n}\left|\frac{(-1)^{k+1} \operatorname{det} A_{1, k}(t)}{\operatorname{det} A(t)}\right| \\
& =\varepsilon\left\|A^{-1}(t)\right\|_{\infty},
\end{aligned}
$$

and hence the proof is completed.

The inequality of Theorem 2 is termed stability inequality in the technical literature. In our setting, contrary to "real" stability problems, now the corresponding equation has a unique solution with a representation formula. This is the reason why we speak about error estimation instead of stability. 
As we pointed out in the Introduction, (1) is related to some investigations of Babbage in sense that finite groups of substitutions are considered. However, the pioneer work of Babbage still motivates and influences the research on Iterative Functional Equations intensively. To illustrate this fact, not claiming completeness, we refer here the papers of Jarczyk [10], Solarz [19], and Zdun [20]. For a comprehensive view of this filed, both for results and open problems, consult the survey of Baron-Jarczyk [4]. For those ones who are interested in the Theory of Functional Equations in a Single Variable, we refer the books of Kuczma [12] and [14]. The books of Aczél [1] and Kuczma [13] subsume the classical results of the Theory of Functional Equations presenting also interesting historical remarks.

Acknowledgements. The authors wish to express their gratitude to ZSOLT PÁLES (University of Debrecen, Hungary) for valuable comments on the paper.

\section{REFERENCES}

[1] J. Aczél, Lectures on Functional Equations and Their Applications, Mathematics in Science and Engineering, vol. 19, Academic Press, New York-London, 1966.

[2] Ch. Babbage, An essay towards a calculus of functions I., Phil. Trans. 105 (1815), 389-423.

[3] Ch. Babbage, An essay towards a calculus of functions II., Phil. Trans. 106 (1816), 179-256.

[4] K. Baron and W. Jarczyk, Recent results on functional equations in a single variable, perspectives and open problems, Aequat. Math. 61 (2001), no. 1-2, 1-48.

[5] M. Bessenyei, Functional equations and finite groups of substitutions, Amer. Math. Monthly 117 (2010), no. 10, 921-927.

[6] M. Bessenyei and Cs. G. Kézi, Functional equations and group substitutions, Linear Algebra Appl. 434 (2011), no. 6, 1525-1531.

[7] M. Bessenyei, Cs. G. Kézi and G. Horváth, Functional equations on finite groups of substitutions, Expo. Math. (2012), to appear.

[8] V. S. Brodskii and A. K. Slipenko, Functional Equations, Visa Skola, Kiev, USSR, 1986.

[9] J. D. Dixon and B. Mortimer, Permutation groups, Graduate Texts in Mathematics, vol. 163, Springer-Verlag, New York, 1996.

[10] W. Jarczyk, Babbage equation on the circle, Publ. Math. Debrecen 63 (2003), no. 3, 389-400.

[11] A. Kerber, Representations of permutation groups. I, Lecture Notes in Mathematics, Vol. 240, Springer-Verlag, Berlin, 1971.

[12] M. Kuczma, Functional Equations in a Single Variable, Monografie Mat. Vol. 46, Polish Scientific Publishers, Warszawa, 1968.

[13] M. Kuczma, An Introduction to the Theory of Functional Equations and Inequalities. Cauchy's Equation and Jensen's Inequality, 2nd ed. with a preface by A. Gilányi, Birkhäuser Verlag, Basel, Switzerland, 2009.

[14] M. Kuczma, B. Choczewski, and R. Ger, Iterative Functional Equations, Encyclopedia of Mathematics and its Applications, vol. 32, Cambridge University Press, Cambridge, 1990.

[15] S. Presić, Méthode de résolution d'une classe d'équations fonctionnelles linéaries, Univ. Beograd, Publ. Elektrotechn. Fak. Scr. Math. Fiz (1963), no. 119, 21-28.

[16] S. Presić, Sur l'équation fonctionnelle $f(x)=H\left(x, f(x), f\left(\theta_{2} x\right), \ldots, f\left(\theta_{n} x\right)\right)$, Univ. Beograd, Publ. Elektrotechn. Fak. Scr. Math. Fiz (1963), no. 118, 17-20.

[17] W. Rudin, Principles of Mathematical Analysis, McGraw-Hill, New York, 1976.

[18] C. G. Small, Functional Equations and How to Solve Them, Springer Science+Buisiness Media, LLC, New York (USA), 2007.

[19] P. Solarz, On some iterative roots on the circle, Publ. Math. Debrecen 63 (2003), no. 4, 677-692.

[20] M. C. Zdun, On iterative roots of homeomorphisms of the circle, Bull. Polish Acad. Sci. Math. 48 (2000), 203213.

Institute of Mathematics, University of Debrecen, H-4010 Debrecen, Pf. 12, Hungary

E-mail address: besse@science.unideb.hu

E-mail address: kezicsaba@science. unideb.hu 